\title{
DARK MATTER DECAY AND ANNIHILATION IN THE LOCAL UNIVERSE: CLUES FROM FERMI
}

\author{
A. J. Cuesta ${ }^{1}$, T. E. Jeltema ${ }^{2}$, F. Zandanel ${ }^{1}$, S. Profumo ${ }^{3,4}$, F. Prada ${ }^{1,12}$, G. Yepes ${ }^{5}$, A. KLypin ${ }^{6}$, Y. Hoffman ${ }^{7}$, \\ S. Gottlöber ${ }^{8}$, J. Primack $^{3}$, M. A. SÁnchez-Conde ${ }^{9,10}$, And C. Pfrommer ${ }^{11}$ \\ ${ }^{1}$ Instituto de Astrofísica de Andalucía (CSIC), E-18008 Granada, Spain; ajcv@iaa.es, fabio@iaa.es \\ ${ }^{2}$ UCO/Lick Observatories, Santa Cruz, CA 95064, USA; tesla@ucolick.org \\ ${ }^{3}$ Department of Physics, University of California, Santa Cruz, CA 95064, USA \\ ${ }^{4}$ Santa Cruz Institute for Particle Physics, University of California, Santa Cruz, CA 95064, USA \\ ${ }^{5}$ Universidad Autónoma de Madrid, Grupo de Astrofísica, E-28049 Madrid, Spain \\ ${ }^{6}$ Department of Astronomy, New Mexico State University, Las Cruces, NM 88003-0001, USA \\ ${ }^{7}$ Racah Institute of Physics, Hebrew University, Jerusalem 91904, Israel \\ ${ }^{8}$ Astrophysical Institute Potsdam, 14482 Potsdam, Germany \\ ${ }^{9}$ Instituto de Astrofísica de Canarias, E-38200 La Laguna, Tenerife, Spain \\ ${ }^{10}$ Departamento de Astrofísica, Universidad de La Laguna, E-38205 La Laguna, Tenerife, Spain \\ ${ }^{11}$ Canadian Institute for Theoretical Astrophysics, Toronto, ON M5S 3H8, Canada \\ Received 2010 July 19; accepted 2010 November 2; published 2010 December 10
}

\begin{abstract}
We present all-sky simulated Fermi maps of $\gamma$-rays from dark matter (DM) decay and annihilation in the local universe. The DM distribution is obtained from a constrained cosmological simulation of the neighboring large-scale structure provided by the CLUES project. The DM fields of density and density squared are then taken as an input for the Fermi observation simulation tool to predict the $\gamma$-ray photon counts that Fermi would detect in 5 years of an all-sky survey for given DM models. Signal-to-noise ratio (S/N) sky maps have also been obtained by adopting the current Galactic and isotropic diffuse background models released by the Fermi Collaboration. We point out the possibility for Fermi to detect a DM $\gamma$-ray signal in local extragalactic structures. In particular, we conclude here that Fermi observations of nearby clusters (e.g., Virgo and Coma) and filaments are expected to give stronger constraints on decaying DM compared to previous studies. As an example, we find a significant $\mathrm{S} / \mathrm{N}$ in DM models with a decay rate fitting the positron excess as measured by PAMELA. This is the first time that DM filaments are shown to be promising targets for indirect detection of DM. On the other hand, the prospects for detectability of annihilating DM in local extragalactic structures are less optimistic even with extreme cross-sections. We make the DM density and density squared maps publicly available online.
\end{abstract}

Key words: astroparticle physics - dark matter - gamma rays: diffuse background - large-scale structure of universe - methods: numerical

Online-only material: color figures

\section{INTRODUCTION}

A large amount of astrophysical evidence suggests that most of the universe's matter content is in the form of cold dark matter (DM). However, the nature of DM is still one of the most important open questions in modern physics. Many different candidates have been proposed as DM constituents (see Bertone et al. 2005 for a review on candidates and experimental searches), yet for the time being there is no evidence in favor of any model. One of the most studied scenarios is that of weakly interacting massive particles (WIMPs), where $\gamma$-rays are generated as secondary products of WIMP decay or annihilation (e.g., Bertone 2010). Therefore, $\gamma$-ray observations, being a complementary approach to direct searches, are a powerful tool to study the nature of DM.

At present, the Imaging Atmospheric Čerenkov Telescopes (IACTs such as MAGIC, HESS, and VERITAS) together with the recently launched Fermi satellite offer great tools to search for the $\gamma$-ray emission due to DM decay or annihilation in the $\mathrm{MeV}-\mathrm{TeV}$ energy range. The main instrument on board Fermi is the Large Area Telescope (LAT), which is designed to explore

\footnotetext{
12 Visiting research physicist at the Santa Cruz Institute for Particle Physics, University of California, Santa Cruz, CA 95064, USA.
}

the entire $\gamma$-ray sky in the $20 \mathrm{MeV}-300 \mathrm{GeV}$ energy range (Atwood et al. 2009). The Fermi-LAT collaboration already reported some of their results on DM searches, with no detection of DM $\gamma$-ray emission in dwarf spheroidal galaxies (Abdo et al. 2010c), clusters (Ackermann et al. 2010), or spectral features (Abdo et al. 2010a, 2010b). IACTs did not succeed in DM detection either (e.g., Aharonian et al. 2008a; Aliu et al. 2009; Aleksić et al. 2010; Acciari et al. 2010). These studies are focused mainly on DM annihilation.

Despite these negative results, the recent detection of an excess of high-energy $(10-100 \mathrm{GeV})$ positrons over the standard expectation from galactic cosmic-ray models by the PAMELA experiment (Adriani et al. 2009b) triggered an interest in the possibility that these positrons originate from DM in the Milky Way. Interestingly, if DM annihilation or decay is to consistently explain the cosmic-ray electron/positron data recently produced by the PAMELA, Fermi-LAT, and HESS collaborations (Abdo et al. 2009; Aharonian et al. 2008b, 2009; Adriani et al. 2009a, 2010), one is generically forced to consider dominantly leptonic final states to avoid overproducing anti-protons and to generate enough high-energy positrons, and a cross-section that is enhanced over its standard value by $\sim 10^{3}$. In turn, this is an interesting scenario for $\gamma$-ray searches, since hard final state leptons yield an unmistakable hard bremsstrahlung $\gamma$-ray spectrum (Pinzke et al. 2009). 
The aim of this Letter is to study DM induced $\gamma$-ray emission using a constrained local universe cosmological simulation, as would be observed by the Fermi satellite in an all-sky $\gamma$-ray survey for given DM models. In Section 2, we describe the cosmological simulation used to infer the DM distribution in the Local Universe. In Section 3, the Fermi observation simulations and the assumed particle physics models are described. In Section 4, we present the main results from the $\gamma$-ray all-sky maps. We finally discuss the main conclusions of our work in Section 5.

\section{CONSTRAINED SIMULATIONS OF THE LOCAL UNIVERSE}

In order to get a detailed description of the DM density distribution in the Local Universe, we use a high-resolution cosmological simulation box from the CLUES Project ${ }^{13}$. This simulation set provides a realistic local density field which is consistent with the $\Lambda \mathrm{CDM}$ cosmology (see Yepes et al. 2009; Gottlöber et al. 2010 for more details). As we want to study $\gamma$-rays from large structures in the Local Universe such as nearby galaxy clusters, we choose the Box160CR simulation. This is a constrained realization with $1024^{3}$ particles in a cube of $160 \mathrm{~h}^{-1} \mathrm{Mpc}$ on a side which was run using the MPIART cosmological code (Kravtsov et al. 1997; Gottlöber \& Klypin 2008). The initial conditions are set assuming WMAP3 cosmology (with $\Omega_{m}=0.24, \Omega_{\Lambda}=0.76, \Omega_{b}=0.042$, $h=0.73, \sigma_{8}=0.75$, and $n=0.95$ ) and implement the constraints from the observed density field so that it reproduces the observed matter distribution in the local universe on large scales at redshift $z=0$ (Hoffman \& Ribak 1991; Klypin et al. 2003). The massive clusters such as Virgo, Coma, and Perseus, together with the Great Attractor, are well reproduced. However, the final positions of these objects are not exactly at their observed positions, with a typical error around $5 h^{-1} \mathrm{Mpc}$.

This cosmological simulation allows us to produce all-sky maps of the local DM density and density squared, which are proportional to $\gamma$-ray emission due to particle DM decay and annihilation, respectively (to include more distant structures, one could use a box replication technique as in Zavala et al. 2010). We follow the method described in Kuhlen et al. (2008) to compute these luminosities. The flux is proportional to $\sum_{i} m_{p} / 4 \pi d_{i}^{2}$ for decay and $\sum_{i} m_{p} \rho_{i} / 4 \pi d_{i}^{2}$ for annihilation, where $i$ runs from 1 to the number of particles in each pixel, $m_{p}$ is the mass of the simulation particle, $\rho_{i}$ is the density associated with the $i$ th particle, computed using the sphere which contains its 32 nearest neighbors (no smoothing kernel was used), and $d_{i}$ is the distance to the observer. Only particles between $5 h^{-1} \mathrm{Mpc}$ and $80 h^{-1} \mathrm{Mpc}$ from the observer, which is placed at the right distance from the Virgo cluster, are taken into account. A proper description of the density field in the innermost $5 h^{-1} \mathrm{Mpc}$, although affected by random density fluctuations at this scale, would require a higher resolution simulation to be resolved properly (as in Libeskind et al. 2010). Thus this region empty of massive large structures is not considered here. We bin these fluxes in a Cartesian grid with 3600 and 1800 pixels of galactic longitude and latitude, respectively. This corresponds to an angular resolution of roughly $0.1 \mathrm{deg}$ pixel $^{-1}$, reproducing the best angular resolution that Fermi has at its highest accessible energy range.

13 http://clues-project.org

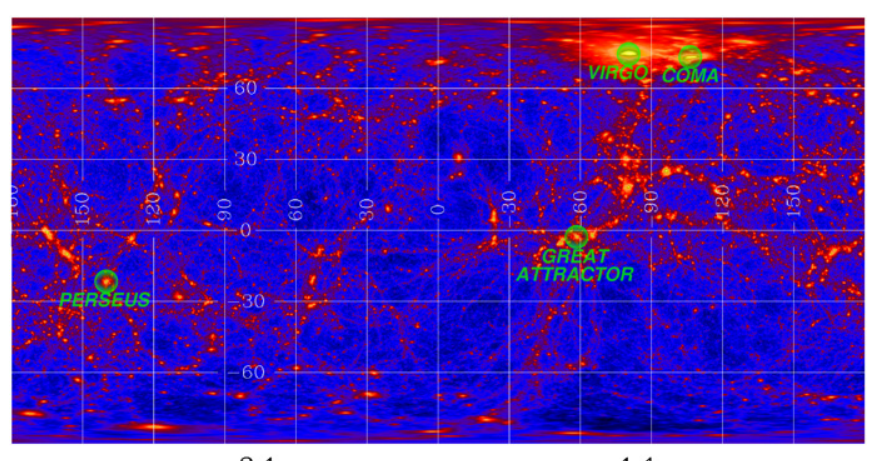

$-3.1 \longrightarrow$

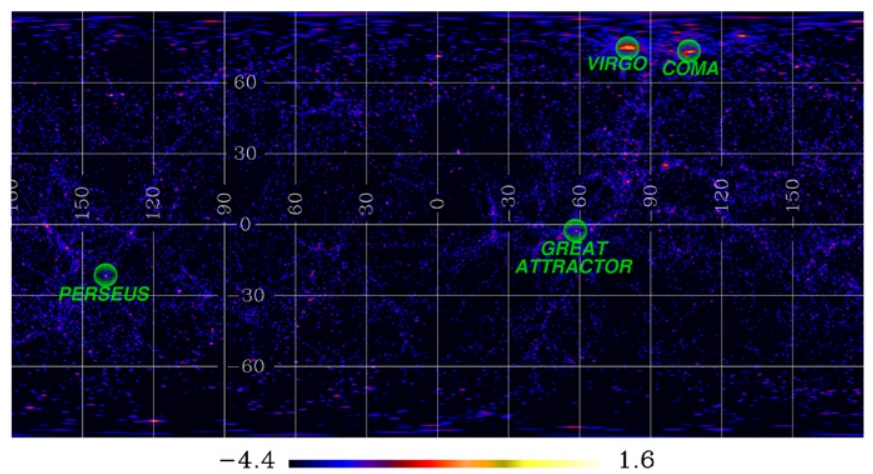

Figure 1. DM distribution in the local universe constrained cosmological simulation Box 160CR. These all-sky maps are Cartesian projections in Galactic coordinates. Top panel shows the density distribution, whereas the bottom panel displays the distribution of density squared. The maps are color-coded according to the $\log _{10}$ of the DM flux, and units are $\mathrm{GeV} / \mathrm{c}^{2} \mathrm{~cm}^{-3} \mathrm{kpc} \mathrm{sr}^{-1}$ for decay map and $\mathrm{GeV}^{2} / \mathrm{c}^{4} \mathrm{~cm}^{-6} \mathrm{kpc} \mathrm{sr}^{-1}$ for the annihilation map. Large structures reproduced by the simulation such as Virgo, Coma, and Perseus clusters, together with the Great Attractor, are labeled. High-resolution versions of these figures are available at http://www.clues-project.org/articles/darkmattermaps.html.

(A color version of this figure is available in the online journal.)

Due to the finite resolution of the simulation, we cannot resolve the very inner center of DM halos. For this reason, we correct the flux, if underestimated, in every pixel where the centers of DM halos lie (see Kuhlen et al. 2008). We assume a Navarro-Frenk-White (NFW) profile (Navarro et al. 1996) for the inner density profile of these halos and we extrapolate it up to the halo center. The scale radius $r_{s}$ of these halos is calculated from the virial mass-concentration relation in Macciò et al. (2008). Typical corrections do not exceed $\sim 25 \%$ and $\sim 250 \%$ of their original value for decay and annihilation, respectively. We note that no boost factor due to DM substructures, or any other effect (such as adiabatic contraction from baryons or Sommerfeld enhancement), is included in our analysis.

The resulting all-sky maps are shown in Figure 1, where known objects are highlighted. The images are done with HEALPix. $^{14}$ Angular projection used here is equirectangular (plate carrée). Maps are color-coded according to the logarithmic flux in each $0.1 \mathrm{deg}$ pixel, measured in $\mathrm{GeV} / \mathrm{c}^{2} \mathrm{~cm}^{-3} \mathrm{kpc} \mathrm{sr}^{-1}$ for decay and $\mathrm{GeV}^{2} / c^{4} \mathrm{~cm}^{-6} \mathrm{kpc} \mathrm{sr}^{-1}$ for annihilation. These maps are used as input for the Fermi-LAT observation simulations, which we describe in the following section.

\section{FERMI SATELLITE OBSERVATION SIMULATIONS}

Using the full sky DM density and density squared maps, the simulated Fermi-LAT observations are produced using the

$\overline{14}$ http://healpix.jpl.nasa.gov (Górski et al. 2005) 
gtobssim routine, part of the Fermi Science Tools package (v9r15p2), which incorporates the Fermi-LAT effective area and point-spread function (PSF) and their energy dependence. All simulations are run to generate a five year observation in the default scanning mode and using the current release of the LAT instrument response functions (P6_V3_DIFFUSE).

In the present study, we specifically adopt two examples for the $\gamma$-ray spectrum from decay or annihilation of the DM particle, chosen to be representative of more general classes of DM models. The first model features a DM particle with a mass of $1.6 \mathrm{TeV}$ yielding a pair of $\mu^{+} \mu^{-}$(Bergström et al. 2009), which was shown to fit accurately the PAMELA data in Papucci $\&$ Strumia (2010). In this case, $\gamma$-ray emission is produced directly in the final state radiation (FSR) as well as through inverse Compton (IC) scattering of the high energy $e^{+}$and $e^{-}$ produced off of cosmic microwave background (CMB) photons. We include the expected contributions from both FSR and IC (see Ackermann et al. 2010 for details). Given that the energy loss timescales for high-energy electrons and positrons produced by muon decays are much shorter than the diffusion timescales in the structures we consider here, we neglect diffusion, and also calculate the emission of said electrons and positrons via IC upscattering of CMB photons. This yields a significant lowenergy component, extending all the way up to energies relevant to the Fermi telescope (Profumo \& Jeltema 2009). We also consider a second, more conventional model, inspired by what it is expected in, e.g., supersymmetric models with a binolike lightest supersymmetric particle: a $100 \mathrm{GeV}$ neutralino yielding a quark-antiquark pair (of $b$ flavor, for definiteness). The primary source of $\gamma$-rays here is the decay of neutral pions produced in the $b \bar{b}$ hadronization chains.

The Fermi simulations of the $\gamma$-ray signal from DM annihilation (both to $b \bar{b}$ and $\mu^{+} \mu^{-}$) were normalized to a DM flux in the Fermi energy range of $9 \times 10^{-8}$ photons $\mathrm{cm}^{-2} \mathrm{~s}^{-1}$ integrated over the full sky. This was chosen in order to obtain good statistics to compare between different extragalactic structures. For $b \bar{b}$, this flux corresponds to a cross-section of $10^{-23} \mathrm{~cm}^{3} \mathrm{~s}^{-1}$. This cross-section value has already been excluded by current indirect searches with Fermi, PAMELA, and HESS (see, e.g., Cirelli et al. 2010; Papucci \& Strumia 2010). Yet we decided to keep this extreme case for comparison purposes. For $\mu^{+} \mu^{-}$, the same total flux corresponds to an annihilation cross-section of $5.8 \times 10^{-23} \mathrm{~cm}^{3} \mathrm{~s}^{-1}$, which gives a good fit to the PAMELA positron excess. This value is only marginally excluded in Ackermann et al. (2010) if cluster substructures are considered, and also in Papucci \& Strumia (2010) if the Milky Way DM halo follows NFW.

In the case of DM decay as, e.g., in supersymmetry with very weak $R$-parity violation induced by a dimension-6 GUTscale operator to both $b \bar{b}$ and $\mu^{+} \mu^{-}$, we simulated a total Fermi flux over the full sky of $1.5 \times 10^{-6}$ photons $\mathrm{cm}^{-2} \mathrm{~s}^{-1}$, which corresponds to a decay lifetime of $\tau \simeq 10^{26} \mathrm{~s}$ for $b \bar{b}$ and $\tau \simeq 3 \times 10^{26} \mathrm{~s}$ for $\mu^{+} \mu^{-}$. These lifetimes are not currently excluded by other $\gamma$-ray constraints and the latter case gives a good fit to the PAMELA-measured positron fraction excess (e.g., Papucci \& Strumia 2010; Cirelli et al. 2010; Chen et al. 2010; Meade et al. 2010; Zhang et al. 2010).

We also include in the simulations realistic treatments of both Galactic and isotropic diffuse backgrounds. In particular, the $\gamma$-ray emission from the Galaxy is quite variable across the sky, an important consideration when comparing the expected signals from known objects. For example, structures lying at low Galactic latitudes like the Great Attractor will have much
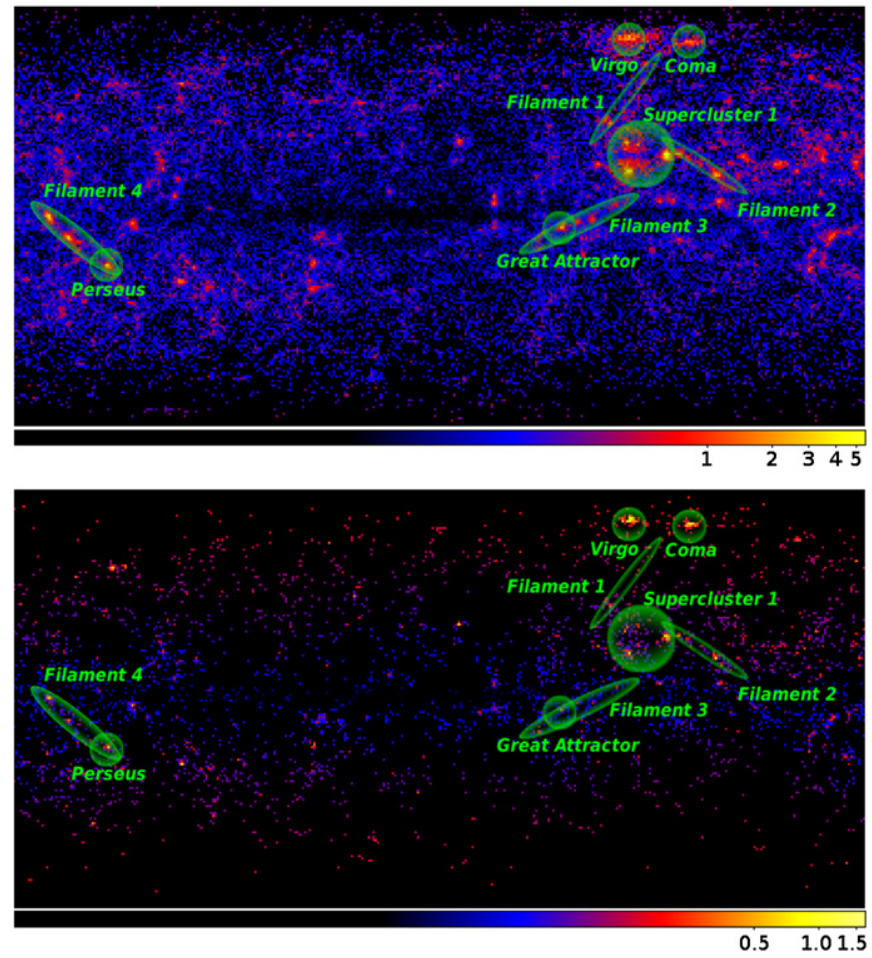

Figure 2. S/N all-sky maps from Fermi simulations for DM $\gamma$-rays in the energy range $100 \mathrm{MeV}-10 \mathrm{GeV}$ built from the Box160CR constrained simulation of the local universe. Results for DM decay (top) and annihilation (bottom) are shown for the $b \bar{b}$ channel model.

(A color version of this figure is available in the online journal.)

higher $\gamma$-ray backgrounds than high latitude objects like the Virgo cluster. We simulated five year Fermi observations of the Galactic and isotropic diffuse backgrounds using the current background models released by the Fermi collaboration ${ }^{15}$ (gll_iem_v02.fit and isotropic_iem_v02.txt, respectively). The output background map is then used to compute signal-to-noise $(\mathrm{S} / \mathrm{N})$ all-sky maps, detailed in the next section.

\section{RESULTS}

In Figure 2, we show our main results: the $\mathrm{S} / \mathrm{N}$ prediction for the extragalactic $\gamma$-ray emission in the $100 \mathrm{MeV}-10 \mathrm{GeV}$ energy range from annihilation and decay of DM in the local universe, as it would be seen by the Fermi satellite after five years of observations. This is the first time that a constrained cosmological simulation is used to generate maps that are consistent with both the currently accepted cosmology and the observed local universe. These maps assume a particle mass of $100 \mathrm{GeV}$ which annihilates or decays through the $b \bar{b}$ channel. Maps for the DM model yielding $\mu^{+} \mu^{-}$are similar but present lower S/N. Pixels are binned in squares of $1 \mathrm{deg}$ which matches the Fermi-LAT PSF at around $1 \mathrm{GeV}$ as well as the typical angular size in the sky of nearby clusters. In order to make a quantitative analysis of DM detectability in large nearby structures, including cluster and filament regions, we computed in Table 1 the photon number counts and $\mathrm{S} / \mathrm{N}$ from annihilation and decay in the $1 \mathrm{GeV}-10 \mathrm{GeV}$ energy range, for both the $b \bar{b}$ and $\mu^{+} \mu^{-}$channels. The $\mathrm{S} / \mathrm{N}$ is defined as the signal over the square root of the signal plus the background $\gamma$-ray emission. This choice of energy range maximizes the

\footnotetext{
15 http://fermi.gsfc.nasa.gov/ssc/data/access/lat/BackgroundModels.html
} 
Table 1

$\mathrm{S} / \mathrm{N}$, Photon Counts, and Backgrounds in Fermi Simulations

\begin{tabular}{|c|c|c|c|c|c|c|c|c|c|}
\hline \multirow[t]{2}{*}{ Object } & \multicolumn{4}{|c|}{$b \bar{b}$ Channel } & \multicolumn{4}{|c|}{$\mu^{+} \mu^{-}$Channel } & \multirow[t]{2}{*}{ Background } \\
\hline & \multicolumn{2}{|c|}{ Ann } & \multicolumn{2}{|c|}{ Dec } & \multicolumn{2}{|c|}{ Ann } & \multicolumn{2}{|c|}{ Dec } & \\
\hline Coma $1 \mathrm{deg}$ & 2.981 & $(20)$ & 2.981 & (20) & 0.567 & (3) & 1.078 & (6) & 25 \\
\hline Coma 5 deg & 1.542 & $(39)$ & 4.976 & $(135)$ & 0.203 & (5) & 1.542 & (39) & 601 \\
\hline Virgo $1 \mathrm{deg}$ & 2.739 & (15) & 3.133 & (18) & 0.000 & (0) & 0.485 & (2) & 15 \\
\hline Virgo 2 deg & 2.858 & $(28)$ & 5.371 & $(61)$ & 0.471 & (4) & 1.444 & (13) & 68 \\
\hline Virgo $5 \mathrm{deg}$ & 1.818 & $(42)$ & 7.700 & $(203)$ & 0.269 & (6) & 2.189 & $(51)$ & 492 \\
\hline Perseus 1 deg & 1.144 & (12) & 5.642 & $(74)$ & 0.493 & (5) & 1.841 & (20) & 98 \\
\hline Perseus 2 deg & 0.700 & (14) & 5.646 & (128) & 0.253 & (5) & 1.518 & (31) & 386 \\
\hline Perseus 5 deg & 0.386 & $(20)$ & 4.542 & $(245)$ & 0.116 & (6) & 1.187 & (62) & 2665 \\
\hline Great Attractor $1 \mathrm{deg}$ & 0.280 & $(8)$ & 2.686 & $(80)$ & 0.105 & (3) & 0.935 & (27) & 807 \\
\hline Great Attractor 2 deg & 0.211 & (13) & 2.581 & $(162)$ & 0.049 & (3) & 0.696 & (43) & 3777 \\
\hline Great Attractor 5 deg & 0.136 & $(23)$ & 2.157 & $(367)$ & 0.041 & (7) & 0.538 & (91) & 28572 \\
\hline Filament $1, d=65 \mathrm{Mpc} h^{-1}$ & 0.224 & (14) & 4.515 & (292) & 0.128 & (8) & 1.348 & (85) & 3891 \\
\hline Filament $2, d=40 \mathrm{Mpc} h^{-1}$ & 0.752 & (67) & 9.317 & (871) & 0.191 & (17) & 2.589 & $(233)$ & 7869 \\
\hline Filament $3, d=65 \mathrm{Mpc} h^{-1}$ & 0.351 & $(84)$ & 4.862 & $(1174)$ & 0.121 & (29) & 1.181 & $(283)$ & 57127 \\
\hline Filament $4, d=55 \mathrm{Mpc} h^{-1}$ & 0.576 & (91) & 8.380 & (1358) & 0.184 & (29) & 2.065 & (328) & 24904 \\
\hline Supercluster $1, d=45 \mathrm{Mpc} h^{-1}$ & 0.911 & (144) & 12.598 & (2066) & 0.254 & (40) & 3.334 & $(531)$ & 24829 \\
\hline
\end{tabular}

Notes. The $\mathrm{S} / \mathrm{N}$ and number of photon counts (in brackets) in the $1 \mathrm{GeV}-10 \mathrm{GeV}$ energy range for our different DM models. For cluster regions, three different radii are considered $(1,2$, and $5 \mathrm{deg}$ ). Filaments $1-4$ represent elongated regions connected to these clusters which are potentially interesting due to their high $\mathrm{S} / \mathrm{N}$. Median distance of halos belonging to these filaments is indicated. Supercluster 1 is a collection of massive halos which accidentally lie along the line of sight. Background counts from the Galactic plus extragalactic diffuse in the same regions are also listed. Note that the annihilation to $b \bar{b}$ case is shown for comparison purposes only.

$\mathrm{S} / \mathrm{N}$ ratio as compared to the $100 \mathrm{MeV}-10 \mathrm{GeV}$ range, as the Fermi-LAT sensitivity is significantly worse at lower energies.

In the case of DM decay, we find that nearby clusters and filamentary regions could be detected for decay lifetimes longer than those currently ruled out by other $\gamma$-ray constraints as discussed above. This shows that extragalactic structures are excellent targets to search for a signal or to place constraints on DM decay models, including those fitting the PAMELA positron data. It is important to note that according to Table 1 the most promising clusters for DM studies are high galactic latitude objects, like Virgo and Coma. The former, however, has not been used in the recent Fermi search for DM in clusters, due to the presence of M87, which is a powerful $\gamma$-ray source, which makes the extraction of a signal or a limit much more complicated than for other clusters. Moreover, we find that $\mathrm{S} / \mathrm{N}$ is not very sensitive to the area of the region under analysis, provided that the aperture radius is no more than few degrees, where the signal saturates and therefore the background noise makes the $\mathrm{S} / \mathrm{N}$ decrease.

We also highlight that, in the case of DM decay, the filamentary structure of the cosmic web constitutes an interesting target for DM searches. To our knowledge, this is the first time that filaments have been considered as targets for DM searches. In this case the $\gamma$-ray luminosity is just proportional to the enclosed mass, whereas this is only approximately true for annihilation (Pinzke et al. 2009). This means that massive extragalactic objects offer the best chance for detection (see Table 1). Large filaments of DM match and even exceed the values of $\mathrm{S} / \mathrm{N}$ as compared to those in large clusters, although caution should be taken regarding the exact orientation in the sky of these filaments as we find some variation in smaller volume-constrained simulations. Superclusters such as the region marked in Figure 2 show even more significant values. Hence, these features of the large-scale structure of the Universe may prove to be a very promising novel way to detect decaying DM with Fermi.

\section{DISCUSSION AND CONCLUSION}

In this Letter, we have presented simulated Fermi maps of extragalactic $\gamma$-rays coming from DM annihilation and decay in the local universe. The DM distribution is taken from a constrained cosmological simulation of the local universe Box160CR by the CLUES project, and it is available online at the following URL: http://www.clues-project.org/articles/ darkmattermaps.html. This distribution is then taken as an input for the Fermi observation simulation to obtain the allsky distribution of $\gamma$-ray photon counts that would be measured by Fermi in a five year survey. Galactic and isotropic $\gamma$-ray diffuse backgrounds are also taken into account. This allows us to get $\mathrm{S} / \mathrm{N}$ all-sky maps to estimate the possibility of detection of $\gamma$-rays from DM in such a survey. We adopted two different particle physics models: a DM particle annihilating (decaying) primarily to a $b \bar{b}$ final state, and a DM model that gives a good fit to the local positron fraction measured by PAMELA and the total electron spectrum measured by Fermi with annihilation (decay) to a $\mu^{+} \mu^{-}$final state.

In the case of DM decay, we find for these models that large clusters with high galactic latitudes, like Virgo and Coma, offer the best chance to be detected together with filamentary regions and large superclusters. Besides, we find that the $\mathrm{S} / \mathrm{N}$ is not a strong function of the area of the analyzed region, which allows for considering large apertures without significant penalty in the results by masking away $\gamma$-ray point sources. This is an important result, as the Fermi Collaboration and other authors have started to severely constrain models of annihilating DM (Ackermann et al. 2010; Abdo et al. 2010a, 2010b, 2010c) 
while decaying DM has comparatively received considerably smaller attention (however, see, Hütsi et al. 2010). Currently available $\gamma$-ray observations provide less stringent constraints in this case, and from a theoretical standpoint, decaying DM is a generic prediction of many theories beyond the Standard Model of particle physics, for both neutralino DM and models that explain the PAMELA positron excess.

We do not find any strong arguments in favor of a possible Fermi detection of extragalactic $\gamma$-rays induced by DM annihilation. However, we cannot exclude completely this possibility since we are not considering possible boost factors from DM substructures, adiabatic compression, and Sommerfeld effect that may significantly enhance the final $\gamma$-ray emission. Besides, the predicted signals will be enhanced due to recent determinations of the cosmological parameter $\sigma_{8}$ suggesting a higher value than the one assumed here, which is consistent with WMAP3 cosmology.

These conclusions should be complemented by an analysis of the galactic components, mainly from DM subhalos like those hosting the DM-rich dwarf spheroidal galaxies around the Milky Way. This has been recently addressed in Anderson et al. (2010) based on the analysis of a high-resolution simulation of a galactic DM halo, as in previous papers by Kuhlen et al. (2008) and Springel et al. (2008). We note that the presence of any galactic foregrounds not modeled here has a potential effect on the significance of our predictions, although only the Galactic center and massive subhalos have been shown in these papers to be relevant. Moreover, the annihilation or decay of DM in Galactic subhalos will produce $\gamma$-ray photons similar to those from DM in local extragalactic structures and thus if these happened to be coincident in the sky it would only enhance the signal. Nevertheless, a spectral confirmation of the potential DM signal is necessary to validate any claim of detection. On the other hand, these results on DM search will benefit from additional hints from the study of the angular power spectrum of the $\gamma$-ray flux (e.g., Fornasa et al. 2009; Hensley et al. 2010; see also Zavala et al. 2010 for a similar approach to that presented here, extended to more distant contributions). An analysis of the anisotropies in the extragalactic diffuse radiation from DM using constrained simulations will be presented elsewhere.

Concluding, we find that Fermi will be able to place strong constraints on the nature of DM by studying extragalactic objects, in particular for decay. The theoretical predictions from constrained simulations should provide the astroparticle community with the most interesting prospects for the detection of the elusive DM particle.

We acknowledge the support of Spanish MICINNs Consolider-Ingenio 2010 Programme MULTIDARK CSD200900064 and ASTROMADRID (S2009/ESP-146). A.J.C. acknowledges the support from MEC Spanish grant FPU AP20051826. G.Y. acknowledges the support of MICINN research grants FPA2009-08958 and AYA2009-13875-C03-02. S.P. is supported by NASA, DoE, and NSF. Y.H. is supported by Israel Science Foundation (13/08). S.G. acknowledges the sup- port of DAAD through PPP program. BOX160CR simulation has been performed at Leibniz Rechenzentrum Munich (LRZ). Fermi-LAT simulation tools were provided by the Fermi-LAT collaboration and the Fermi Science Support Center.

\section{REFERENCES}

Abdo, A. A., et al. 2009, Phys. Rev. Lett., 102, 181101

Abdo, A. A., et al. 2010a, J. Cosmol. Astropart. Phys., JCAP04(2010)014

Abdo, A. A., et al. 2010b, Phys. Rev. Lett., 104, 091302

Abdo, A. A., et al. 2010c, ApJ, 712, 147

Acciari, V. A., et al. 2010, ApJ, 720, 1174

Ackermann, M., et al. 2010, J. Cosmol. Astropart. Phys., JCAP05(2010)025

Adriani, O., et al. 2009a, Phys. Rev. Lett., 102, 051101

Adriani, O., et al. 2009b, Nature, 458, 607

Adriani, O., et al. 2010, Phys. Rev. Lett., 105, 121101

Aharonian, F., et al. 2008a, Astropart. Phys., 29, 55

Aharonian, F., et al. 2008b, Phys. Rev. Lett., 101, 261104

Aharonian, F., et al. 2009, A\&A, 508, 561

Aleksić, J., et al. 2010, ApJ, 710, 634

Aliu, E., et al. 2009, ApJ, 697, 1299

Anderson, B., Kuhlen, M., Johnson, R., Madau, P., \& Diemand, J. 2010, ApJ, 718,899

Atwood, W. B., et al. 2009, ApJ, 697, 1071

Bergström, L., Edsjö, J., \& Zaharijas, G. 2009, Phys. Rev. Lett., 103, 031103

Bertone, G. (ed.) 2010, Particle Dark Matter: Observations, Models and Searches (Cambridge: Cambridge Univ. Press)

Bertone, G., Hooper, D., \& Silk, J. 2005, Phys. Rep., 405, 279

Chen, C., Mandal, S. K., \& Takahashi, F. 2010, J. Cosmol. Astropart. Phys., JCAP01(2010)023

Cirelli, M., Panci, P., \& Serpico, P. D. 2010, Nucl. Phys. B, 840, 284

Fornasa, M., Pieri, L., Bertone, G., \& Branchini, E. 2009, Phys. Rev. D, 80, 023518

Górski, K. M., Hivon, E., Banday, A. J., Wandelt, B. D., Hansen, F. K., Reinecke, M., \& Bartelmann, M. 2005, ApJ, 622, 759

Gottlöber, S., Hoffman, Y., \& Yepes, G. 2010, in High Performance Computing in Science and Engineering, ed. S. Wagner et al. (Berlin: Springer), 309

Gottlöber, S., \& Klypin, A. 2008, in High Performance Computing in Science and Engineering, ed. S. Wagner et al. (Berlin: Springer), 29

Hensley, B. S., Siegal-Gaskins, J. M., \& Pavlidou, V. 2010, ApJ, 723, 277

Hoffman, Y., \& Ribak, E. 1991, ApJ, 380, L5

Hütsi, G., Hektor, A., \& Raidal, M. 2010, J. Cosmol. Astropart. Phys., JCAP07(2010)008

Klypin, A., Hoffman, Y., Kravtsov, A. V., \& Gottlöber, S. 2003, ApJ, 596, 19

Kravtsov, A. V., Klypin, A. A., \& Khokhlov, A. M. 1997, ApJS, 111, 73

Kuhlen, M., Diemand, J., \& Madau, P. 2008, ApJ, 686, 262

Libeskind, N. I., Yepes, G., Knebe, A., Gottlöber, S., Hoffman, Y., \& Knollmann, S. R. 2010, MNRAS, 401, 1889

Macciò, A. V., Dutton, A. A., \& van den Bosch, F. C. 2008, MNRAS, 391, 1940

Meade, P., Papucci, M., Strumia, A., \& Volansky, T. 2010, Nucl. Phys. B, 831, 178

Navarro, J. F., Frenk, C. S., \& White, S. D. M. 1996, ApJ, 462, 563

Papucci, M., \& Strumia, A. 2010, J. Cosmol. Astropart. Phys., JCAP03(2010)014

Pinzke, A., Pfrommer, C., \& Bergström, L. 2009, Phys. Rev. Lett., 103, 181302

Profumo, S., \& Jeltema, T. E. 2009, J. Cosmol. Astropart. Phys., JCAP07(2009)020

Springel, V., et al. 2008, Nature, 456, 73

Yepes, G., Martínez-Vaquero, L. A., Gottlöber, S., \& Hoffman, Y. 2009, in AIP Conf. Ser. 1178, The CLUES Project: Constrained Local Universe Simulations, ed. C. Balazs \& F. Wang (Melville, NY: AIP), 64

Zavala, J., Springel, V., \& Boylan-Kolchin, M. 2010, MNRAS, 405, 593

Zhang, L., Weniger, C., Maccione, L., Redondo, J., \& Sigl, G. 2010, J. Cosmol. Astropart. Phys., JCAP06(2010)027 


\title{
ERRATUM: "DARK MATTER DECAY AND ANNIHILATION IN THE LOCAL UNIVERSE: CLUES FROM FERMI" (2011, ApJ, 726, L6)
}

\author{
A. J. Cuesta ${ }^{1}$, T. E. Jeltema ${ }^{2,3,4}$, F. Zandanel ${ }^{5}$, S. Profumo ${ }^{3,4}$, F. Prada $^{5,14}$, G. YePes $^{6}$, A. Klypin ${ }^{7}$, Y. Hoffman $^{8}$, \\ S. Gottlöber ${ }^{9}$, J. Primack ${ }^{3}$, M. A. SÁnchez-Conde ${ }^{10,11,12}$, And C. Pfrommer ${ }^{13}$ \\ ${ }^{1}$ Yale Center for Astronomy and Astrophysics, Yale University, New Haven, CT 06511, USA; antonio.cuesta@yale.edu \\ ${ }^{2}$ UCO/Lick Observatories, Santa Cruz, CA 95064, USA; tesla@ucolick.org \\ ${ }^{3}$ Department of Physics, University of California, Santa Cruz, CA 95064, USA \\ ${ }^{4}$ Santa Cruz Institute for Particle Physics, University of California, Santa Cruz, CA 95064, USA \\ ${ }^{5}$ Instituto de Astrofísica de Andalucía (CSIC), E-18008 Granada, Spain; fabio@iaa.es \\ ${ }^{6}$ Universidad Autónoma de Madrid, Grupo de Astrofísica, E-28049 Madrid, Spain \\ ${ }^{7}$ Department of Astronomy, New Mexico State University, Las Cruces, NM 88003-0001, USA \\ ${ }^{8}$ Racah Institute of Physics, Hebrew University, Jerusalem 91904, Israel \\ ${ }^{9}$ Astrophysical Institute Potsdam, 14482 Potsdam, Germany \\ ${ }^{10}$ Instituto de Astrofísica de Canarias, E-38200 La Laguna, Tenerife, Spain \\ ${ }^{11}$ Departamento de Astrofísica, Universidad de La Laguna, E-38205 La Laguna, Tenerife, Spain \\ ${ }^{12}$ SLAC National Laboratory and Kavli Institute for Particle Astrophysics and Cosmology, Menlo Park, CA 94025, USA \\ ${ }^{13}$ Heidelberg Institute for Theoretical Studies, Schloss-Wolfsbrunnenweg 35, D-69118 Heidelberg, Germany \\ Received 2011 December 31; published 2012 January 17
}

Online-only material: color figure

The authors corrected a mistake in the computation of the area assigned to each pixel in the signal maps, which was missing a factor of $0.5 \pi \cos (b)$. While this error is not very important at mid-latitudes, the area of the pixels at the Galactic equator is $57 \%$ larger than previously estimated (hence lowering the signal), and is a factor of 2.5 smaller at the position of Virgo $\left(b \simeq 75^{\circ}\right)$, increasing the previously estimated signal arbitrarily as we approach to the Galactic poles. The signal-to-noise $(\mathrm{S} / \mathrm{N})$ values of Table 1 , as well as the maps in Figure 2 of the published version of the paper, are affected, and we report below the corrected version of both the table and the figure (Table 1 and Figure 1, respectively). Note that the dark matter density and density-squared maps of Figure 1 of the published version of the paper are not affected by this error. The conclusions in the original paper remain valid and the values of the $\mathrm{S} / \mathrm{N}$ for the high-latitude objects we focused on in the paper (mainly Virgo and Coma) are now even stronger.

\footnotetext{
${ }^{14}$ Visiting research physicist at the Santa Cruz Institute for Particle Physics, University of California, Santa Cruz, CA 95064, USA.
}

Table 1

$\mathrm{S} / \mathrm{N}$, Photon Counts, and Backgrounds in Fermi Simulations

\begin{tabular}{|c|c|c|c|c|c|c|c|c|c|}
\hline \multirow{3}{*}{$\begin{array}{l}\text { Object } \\
\text { Coma } 1 \mathrm{deg}\end{array}$} & \multicolumn{4}{|c|}{$b \bar{b}$ Channel } & \multicolumn{4}{|c|}{$\mu^{+} \mu^{-}$Channel } & \multirow{3}{*}{$\begin{array}{c}\text { Background } \\
25\end{array}$} \\
\hline & \multicolumn{2}{|c|}{ ann } & \multicolumn{2}{|c|}{ dec } & \multicolumn{2}{|c|}{ ann } & \multicolumn{2}{|c|}{ dec } & \\
\hline & 5.297 & (44) & 5.297 & (44) & 1.237 & (7) & 2.109 & (13) & \\
\hline Coma 2 deg & 5.068 & (68) & 7.583 & (114) & 0.818 & (9) & 2.741 & (33) & 112 \\
\hline Coma 5 deg & 3.245 & (85) & 10.078 & (303) & 0.445 & (11) & 3.245 & (85) & 601 \\
\hline Virgo $1 \mathrm{deg}$ & 5.041 & (36) & 5.646 & (43) & 0.000 & $(0)$ & 1.118 & (5) & 15 \\
\hline Virgo 2 deg & 5.831 & (68) & 10.025 & (147) & 1.132 & (10) & 3.116 & (31) & 68 \\
\hline Virgo $5 \mathrm{deg}$ & 4.185 & (102) & 15.588 & (488) & 0.666 & (15) & 5.068 & (126) & 492 \\
\hline Perseus 1 deg & 0.777 & (8) & 4.178 & $(51)$ & 0.298 & (3) & 1.323 & (14) & 98 \\
\hline Perseus 2 deg & 0.503 & (10) & 4.042 & (88) & 0.152 & (3) & 1.041 & (21) & 386 \\
\hline Perseus $5 \mathrm{deg}$ & 0.271 & (14) & 3.156 & (168) & 0.077 & (4) & 0.807 & (42) & 2665 \\
\hline GAttractor $1 \mathrm{deg}$ & 0.175 & (5) & 1.741 & $(51)$ & 0.070 & (2) & 0.592 & (17) & 807 \\
\hline GAttractor 2 deg & 0.130 & (8) & 1.654 & (103) & 0.033 & (2) & 0.438 & (27) & 3777 \\
\hline GAttractor $5 \mathrm{deg}$ & 0.089 & (15) & 1.379 & (234) & 0.024 & (4) & 0.343 & (58) & 28572 \\
\hline Filament $1, d=65 \mathrm{Mpc} h^{-1}$ & 0.224 & (14) & 4.485 & (290) & 0.112 & (7) & 1.379 & (87) & 3891 \\
\hline Filament2, $d=40 \mathrm{Mpc} h^{-1}$ & 0.517 & (46) & 6.541 & $(602)$ & 0.135 & (12) & 1.797 & (161) & 7869 \\
\hline Filament $3, d=65 \mathrm{Mpc} h^{-1}$ & 0.226 & (54) & 3.117 & $(750)$ & 0.079 & (19) & 0.756 & $(181)$ & 57127 \\
\hline Filament $4, d=55 \mathrm{Mpc} h^{-1}$ & 0.380 & $(60)$ & 5.486 & $(881)$ & 0.120 & (19) & 1.338 & $(212)$ & 24904 \\
\hline Supercluster1, $d=45 \mathrm{Mpc} h^{-1}$ & 0.640 & (101) & 8.915 & (1445) & 0.177 & (28) & 2.343 & (372) & 24829 \\
\hline
\end{tabular}

Notes. The $\mathrm{S} / \mathrm{N}$ and number of photon counts (in brackets) in the $1-10 \mathrm{GeV}$ energy range for our different DM models. For cluster regions, three different radii are considered $\left(1^{\circ}, 2^{\circ}\right.$, and $\left.5^{\circ}\right)$. Filaments $1-4$ represent elongated regions connected to these clusters which are potentially interesting due to their high S/N. Median distance of halos belonging to these filaments is indicated. Supercluster1 is a collection of massive halos which accidentally lie along the line of sight. Background counts from the Galactic plus extragalactic diffuse in the same regions are also listed. Note that the annihilation to $b \bar{b}$ case is shown for comparison purposes only. 

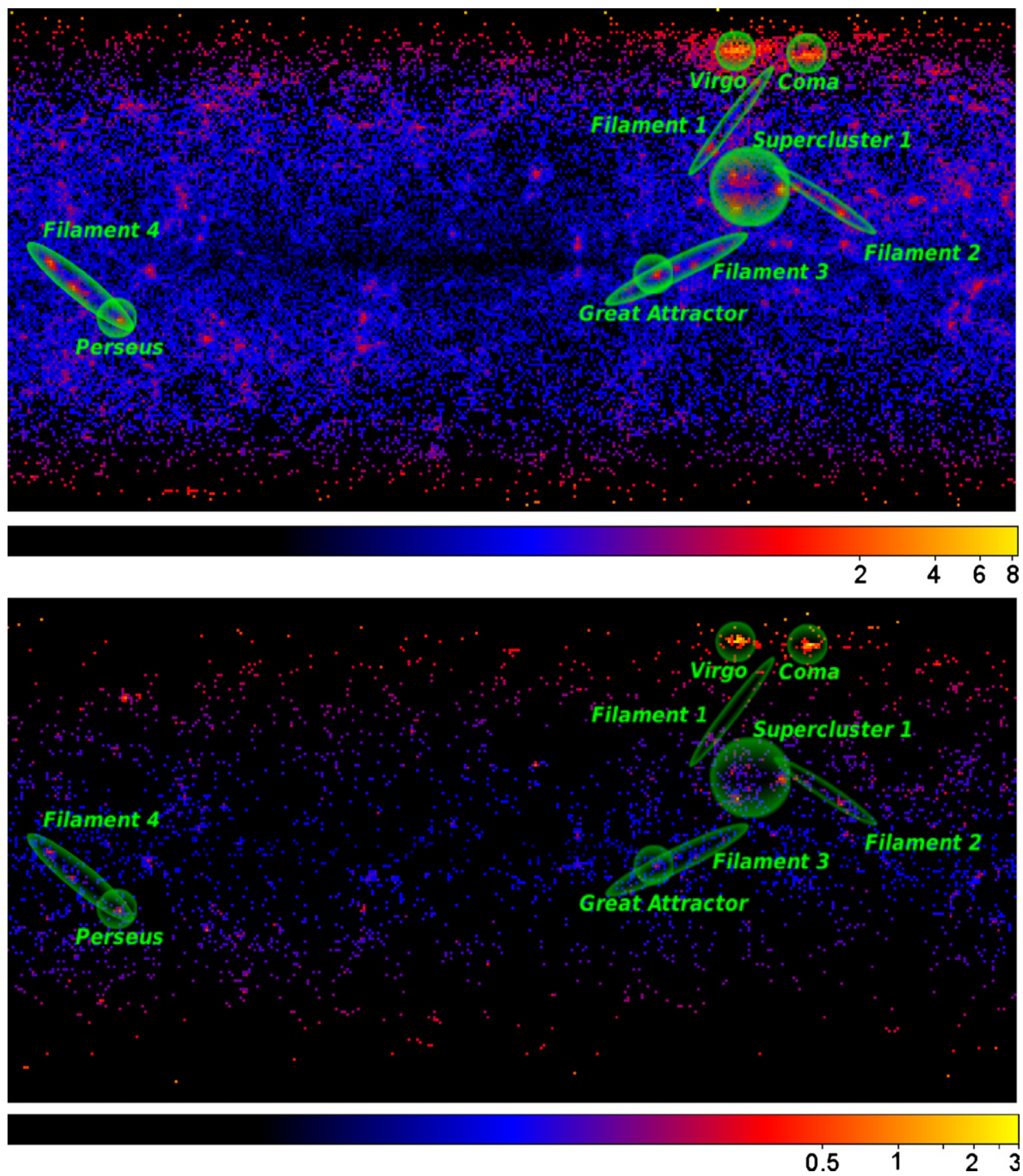

Figure 1. S/N all-sky maps from Fermi simulations for DM $\gamma$-rays in the energy range $100 \mathrm{MeV}-10 \mathrm{GeV}$ built from the Box $160 \mathrm{CR}$ constrained simulation of the local universe. Results for DM decay (top) and annihilation (bottom) are shown for the $b \bar{b}$ channel model.

(A color version of this figure is available in the online journal.)

The updated FITS files for the dark matter density and density-squared maps are available online at: http://www.clues-project.org/ articles/darkmattermaps.html.

We thank the support of Spanish MICINNs Consolider-Ingenio 2010 Programme MULTIDARK CSD2009-00064 and ASTROMADRID (S2009/ESP-146). A.J.C. thanks support from MEC Spanish grant FPU AP2005-1826. G.Y. acknowledges support of MICINN research grants FPA2009-08958 and AYA2009-13875-C03-02. S.P. is supported by NASA, DoE, and NSF. Y.H. is supported by Israel Science Foundation (13/08). S.G. acknowledges support of DAAD through PPP program. BOX160CR simulation has been performed at Leibniz Rechenzentrum Munich (L.R.Z.). Fermi-LAT simulation tools were provided by the FermiLAT collaboration and the Fermi Science Support Center. A.J.C. thanks the corresponding authors of Gómez-Vargas et al. 2011 for realizing this error when doing the analysis of their signal-to-noise maps.

\section{REFERENCE}

Gómez-Vargas, G. A., Fornasa, M., Zandanel, F., et al. 2011, JCAP, in press (arXiv:1110.3305) 